\title{
A Novel Method for Tumor Localization and Tracking in Radiation Therapy
}

\author{
Mohammad Pourhomayoun, Mark Fowler and Zhanpeng Jin \\ Dept. of Electrical and Computer Engineering, Binghamton Univ., Binghamton, NY, USA, 13902-6000, \\ Phone: 607-777-6973, mpourho1@binghamton.edu,mfowler@binghamton.edu, zjin@binghamton.edu.
}

\begin{abstract}
Because the position of a tumor changes during radiation therapy (because of respiration or patient movements), real-time tumor tracking is necessary during radiation therapy in order to deliver a sufficient dose of radiation to the tumor without damaging the surrounding healthy tissues. In this paper, we propose a novel tumor positioning method based on spatial sparsity. We estimate the position by processing the received signals from only one implantable RF transmitter. The method is easier to implement, non-iterative, faster and more accurate compared to common magnetic transponder based methods. We evaluate the performance of the proposed method using MonteCarlo simulation.
\end{abstract}

\section{INTRODUCTION}

Radiation therapy is an effective method to combat cancerous tumors by delivering high doses of ionizing radiation to a tumor to kill or control the growth of malignant cells [1]. Intensity-Modulated Radiation Therapy (IMRT) has greatly increased the ability to deliver an accurate radiation dose to target volumes. Knowing the accurate position of the tumor is a very important issue in radiation therapy, because any small motion in the position of the tumor causes the radiation to be delivered to the surrounding healthy tissues rather than the tumor area, which not only degrades the performance of the treatment because of lack of sufficient dose for the tumor, but also it may cause severe side effects such as secondary cancer [2],[3]. Since the position of the tumor changes during radiation therapy (because of respiration, gastro-intestinal, cardiac system and circulation or even patient movements), real-time tumor tracking is necessary in radiation therapy treatments in order to deliver an accurate amount of radiation to the tumor without damaging the surrounding healthy tissues [4], [2].

There are several methods proposed in the literature for tumor tracking in IMRT treatments based on implanting several wired or wireless devices (called beacons) inside or in the vicinity of the tumor [2]-[13]. The Calypso Localization system is one of the most prevalent methods which is widely used for tumor positioning in prostate radiation therapy [6], [7],[8],[3]. In the Calypso system, three magnetic transponders are implanted in or near the target. Localization of the transponders is achieved using an electromagnetic array consisting of four electromagnetic coils to excite the transponders and 32 receiving coils to pick up the response signal coming from the transponders. The positions of the implanted transponders are calculated relative to the magnetic array based on the response measurements [6], [3]. There are several other electromagnetic tracking systems (such as in [2], [13]) that use a similar idea to track the tumor position during the radiation therapy.

In this paper, we propose a novel positioning method based on spatial sparsity in 3D space to achieve more accurate results. The system is faster compared to common magnetic tracking methods since there is no need for transponder excitation and also frequently switching of the magnetic array between the transmit and receive modes.

In the proposed method, we use only one wireless implantable RF transmitter (which has the potential to be smaller than the magnetic transponders because there is no need for RLC resonance circuit) implanted inside or in the vicinity of the tumor. The transmitter (i.e implant) emits an RF signal and the position will be determined by processing the received signals by a sensor array. The positioning is based on both time-of-arrival (TOA) and received signal strength (RSS) exploiting the spatial sparsity of the emitter in the 3D space. Contrary to classic TOA or RSS based localization methods, we use convex optimization theory to estimate the location of the emitter directly without going through the intermediate stage of TOA or RSS estimation. Since we don't need to estimate the time-of-arrival or signal strength for each one of the sensors by its own in a separate stage, and we also exploit the spatial sparsity to estimate the emitter location directly, this method is much more accurate in positioning and very robust to multipath conditions (caused by signal reflections at the boundaries of body organs) compared to classic TOA/RSS based methods.

It is obvious that in emitter localization problems, the number of emitters is much smaller than the number of all grid points in a fine grid on the $x-y$ plane or $x-y-z$ space. Thus, imagine a sparse grid matrix that has a positive number at each one of the grid points containing an emitter (i.e implant) and zeros in the rest of the grid points; this can be reformed as a sparse vector. In this context, a sparse vector is a vector containing only a small number of non-zero elements. Since each element of this grid vector corresponds to one grid point in the $x-y-z$ space, we can estimate the location of emitter(s) by extracting the position of the non-zero elements of the sparsest vector that satisfies the delay and path lost relationship between transmitted signals and received signals. Thus, after formulating the problem in terms of the sparse grid 
vector, we can estimate this vector by pushing sparsity using $\ell_{1}$-norm minimization (which is a convex optimization problem) on the grid vector, subject to the delay and signal strength relationship between the signals transmitted from the grid points and the signals received by the sensors [14][15].

The human body includes various organs with different types of tissue. Since the electrical characteristics of the body - such as the relative permittivity - varies for different tissue types, the signal propagation velocity (which is expressed as a function of the relative permittivity) and consequently the time-of-arrival highly depends on the tissue where the signal passes through [16][14][16]. The power absorption parameters and path loss exponent also vary by thickness of the tissue [17]. Because of these reasons, the traditional in-body localization methods based on RSS or TOA are very challenging and sometimes inaccurate unless we have some prior information about the location of the implant. Even if this a priori information is available it is hard to exploit it in the classical location methods.

However, in this paper we develop a novel tissue-adaptive one-stage method based on both time-of-arrival and path loss. In this method, we consider the propagation velocity and path loss exponent as location-dependent parameters that can be exploited to estimate the implant location more precisely. We take advantage of spatial sparsity of the emitter in the 3D space to estimate the emitter location directly without going through the intermediate stage of TOA or path loss estimation.

\section{PROBLEM FORMULATION}

Suppose that an emitter (the implanted RF beacon) transmits a signal and $L$ sensors receive that signal. The complex baseband signal observed by the $l^{\text {th }}$ sensor is

$$
r_{l}(t)=\alpha_{l} s\left(t-\tau_{l}\right)+n_{l}(t)
$$

where $s(t)$ is the transmitted signal, $\tau_{l}$ is the signal delay, $\alpha_{l}$ is the path attenuation, and $n_{l}(t)$ is the noise.

In equation (1), $\alpha_{l}$ represents the path loss in addition to a constant phase shift. The path loss model in $\mathrm{dB}$ is given by [17],

$$
P L(d)=P L\left(d_{0}\right)+10 \beta \log _{10}\left(d / d_{0}\right)+S, \quad d \geq d_{0}
$$

where $P L(d)$ is the path loss at distance $d, P L\left(d_{0}\right)$ is the path loss at the reference distance $d_{0}, \beta$ is the path loss exponent value and $S$ is a zero mean Gaussian random variable (in $\mathrm{dB}$ ) representing the shadowing effect, $S \sim N\left(0, \sigma_{s}^{2}\right)$ [17]. Table I shows the path loss parameters for the implant to body surface model [17].

TABLE I. PATH LOSS PARAMETERS: IMPLANT TO BODY SURFACE MODEL

\begin{tabular}{|c|c|c|c|}
\hline Implant to Body Surface & $\left.\mathbf{P L}\left(\mathbf{d}_{\mathbf{0}}\right) \mathbf{( d B}\right)$ & $\mathbf{B}$ & $\boldsymbol{\sigma}_{\mathbf{s}}(\mathbf{d B})$ \\
\hline Deep Tissue & 47.14 & 4.26 & 7.85 \\
\hline Near Surface & 49.81 & 4.22 & 6.81 \\
\hline
\end{tabular}

In free space, we can easily assume that the signal propagation velocity is constant. However, for localization inside the human body the propagation velocity is not constant and it depends on the tissue where the signal passes through. We can calculate the average relative permittivity and average velocity for the path in which the signal is traveling as follows [16],

$$
\begin{aligned}
& v_{\text {avg }}=\frac{c}{\sqrt{\varepsilon_{a v g}}} \\
& \varepsilon_{a v g}=\sum_{i=1}^{N_{I}} \varepsilon_{i} p_{i},
\end{aligned}
$$

where $v_{a v g}$ is the average propagation velocity, $N_{I}$ is the number of different tissues on the path, $\varepsilon_{i}$ is the relative permittivity of $i^{\text {th }}$ tissue at desired frequency (the values are available for various frequencies and different tissues such as muscle, fat, bone, stomach, intestine and so on [16]) and $p_{i}$ is the percentage of each tissue on the path. We are able to calculate the $v_{a v g}$ for each path using the equation (3) having the tissue configuration of the human body acquired beforehand from an MRI or CT system [16].

Assume that each sensor collects $N_{s}$ signal samples at sampling frequency $F_{s}=1 / T_{s}$. Then we have

$$
\boldsymbol{r}_{l}=\alpha_{l} \boldsymbol{D}_{l} \boldsymbol{s}+\boldsymbol{n}_{l}
$$

where $\quad r_{l} \triangleq\left[r_{l}\left(t_{1}\right), \quad r_{l}\left(t_{2}\right), \quad \ldots \quad, \quad r_{l}\left(t_{N s}\right)\right]^{T} \quad$ is the vector containing $N_{s}$ samples of the received signal by $l^{\text {th }}$ sensor, $s \triangleq\left[s\left(t_{1}\right), \quad s\left(t_{2}\right), \quad \ldots, \quad s\left(t_{N s}\right)\right]^{T}$ is $N_{s}$ samples of the transmitted signal, $\boldsymbol{n}_{l}$ is the noise vector and $\boldsymbol{D}_{l}$ is the time sample shift operator by $k_{l}=\left(\tau_{l} / T_{s}\right)$ samples where $\tau_{l}=\left(d_{l} / v_{\text {avg, },}\right)$ is delay, $d_{l}$ is the distance between emitter and the $l^{\text {th }}$ sensor and $v_{a v g, l}$ is the average velocity on the path from emitter to the $l^{\text {th }}$ sensor derived from (3). We can write $\boldsymbol{D}_{l}=\boldsymbol{D}^{k_{l}}$ where $\boldsymbol{D}$ is an $N_{s} \times N_{s}$ permutation matrix defined as $[\boldsymbol{D}]_{i j}=1$ if $i=j+1,[\boldsymbol{D}]_{0, N-1}=1$ and $[\boldsymbol{D}]_{i j}=0$ otherwise:

$$
\boldsymbol{D}=\left[\begin{array}{cccc}
0 & & & 1 \\
1 & 0 & & \\
& \ddots & \ddots & \\
0 & & 1 & 0
\end{array}\right] \quad ; \quad \boldsymbol{D}_{l}=\left[\begin{array}{cccc}
0 & & & 1 \\
1 & 0 & & \\
& \ddots & \ddots & \\
0 & & 1 & 0
\end{array}\right]^{k_{l}}
$$

To simplify the notations, we assume that we are interested in estimating the location of the target in the two-dimensional $(x-y)$ plane. However, it is easy to expand the localization problem to the three-dimensional case.

Now, we assign a number $\zeta_{x, y}$ to each one of the grid points $(x, y)$. Assume that $\zeta_{x, y}$ is one for the grid points 
containing an emitter (implant) and zero for the rest of the grid points. Thus, the signal vector received by $l^{\text {th }}$ sensor will be

$$
\boldsymbol{r}_{l}=\sum_{x} \sum_{y} \zeta_{x, y} \alpha_{l, x, y} \boldsymbol{D}_{l, x, y} \boldsymbol{s}+\boldsymbol{n}_{l},
$$

where $\boldsymbol{D}_{l, x, y}$ is the time sample shift operator w.r.t sensor $l$ assuming that the emitter is located in the grid point $(x, y)$ and the summations are over all grid points in the desired $(x, y)$ range. Note that $\boldsymbol{D}_{l, x, y}$ and $\alpha_{l, x, y}$ are known in (5) since the location of the sensor $l$ and each grid point $(x, y)$ is known and we are able to find the delay and the path loss from (2) and (3) for the distance from grid point $(x, y)$ to the sensor $l$. The unknown term is $\zeta_{x, y}$ that represents which one of the grid point contains the emitter (i.e implant beacon). Now, if we reform all of the grid points in a column vector and re-arrange the indices, we will have

$$
\boldsymbol{r}_{l}=\sum_{n=1}^{N} \zeta_{n} \alpha_{l, n} \boldsymbol{D}_{l, n} \boldsymbol{s}+\boldsymbol{n}_{l}
$$

Now, we define the matrix $\Lambda_{n}$ as the delay operator w.r.t all $L$ sensors:

$$
\Lambda_{n} \triangleq\left[\begin{array}{llll}
\alpha_{1, n} \boldsymbol{D}_{1, n} & \alpha_{2, n} \boldsymbol{D}_{2, n} & \cdots & \alpha_{L, n} \boldsymbol{D}_{L, n}
\end{array}\right]_{N_{s} \times L N_{s}}
$$

Then, we can define $\boldsymbol{\theta}_{n}, n \in\{1,2, \ldots, N\}$ as an $L N_{s} \times 1$ vector containing all signals received by all $L$ sensors when the emitter is in grid point $n$ as,

$$
\boldsymbol{\theta}_{n} \triangleq \Lambda_{n}{ }^{T} \times \boldsymbol{S}
$$

where $(.)^{\mathrm{T}}$ is the matrix transpose. Now, if we arrange all vectors $\boldsymbol{\theta}_{n}$ for $n: 1 \ldots N$ as the columns of a matrix $\boldsymbol{\Theta}$ as,

$$
\boldsymbol{\Theta}=\left[\begin{array}{llll}
\boldsymbol{\theta}_{1} & \boldsymbol{\theta}_{2} & \ldots & \boldsymbol{\theta}_{N}
\end{array}\right]_{L N_{s} \times N},
$$

then we have

$$
\boldsymbol{r}=\boldsymbol{\Theta} \times \boldsymbol{z}+\boldsymbol{n}
$$

where $\quad \boldsymbol{r} \triangleq\left[\begin{array}{llll}\boldsymbol{r}_{1}^{T} & \boldsymbol{r}_{2}^{T} & \ldots & \boldsymbol{r}_{L}^{T}\end{array}\right]^{T}{ }_{L N_{s} \times 1}$ is the vector of all $L$ received signals, $z \triangleq\left[\begin{array}{lllll}\zeta_{1} & \zeta_{2} & \ldots & \zeta_{N}\end{array}\right]_{N \times 1}^{T}$ is the sparse vector of $z$-values assigned to each grid point and $\boldsymbol{n}$ is the noise.

Now, we can solve our problem by forming the regularized Basis Pursuit Denoising (BPDN) problem as [18]:

$$
\hat{z}=\arg \min \|\boldsymbol{\Theta} \times \boldsymbol{z}-\boldsymbol{r}\|_{2}+\lambda\|\boldsymbol{z}\|_{1}
$$

where $\|\cdot\|_{p}$ is the $\ell_{p}$-norm defined as $\|v\|_{p}=\sqrt[p]{\sum_{i}\left|v_{i}\right|^{p}}$.

\section{SIMULATION RESULTS AND CONCLUSION}

We examined the performance of the proposed method using Monte-Carlo computer simulation with 100 runs each time. Fig. 1-(a) shows a simple pattern for tumor (or implant) movement in X direction. Fig. 1-(b) shows the RMS Error for implant location estimation for the movement pattern in (a). In this simulation $\mathrm{SNR}=10 \mathrm{~dB}$ and we used a sensor array including 16 sensors to receive an RF signal from one implanted emitter. We used a BPSK signal with frequency of $405 \mathrm{MHz}$ and the sampling frequency of $100 \mathrm{MHz}$. The simulation results show the accurate localization and high performance of this method (less than $1.5 \mathrm{~mm}$ error).

In another simulation, we ran Monte-Carlo algorithm for various numbers of sensors $(4,8,12$ and 16 sensors with 100 runs each time), in multipath and shadowing conditions for two different SNRs (0dB and 10dB). In this simulation, the position of the implant has been chosen randomly. Fig. 2 shows the RMS Error vs. number of sensors for estimating the location of the target in $(x, y)$ plane. As we see, the results show that the proposed method has very good performance even for low SNRs and with small number of sensors.

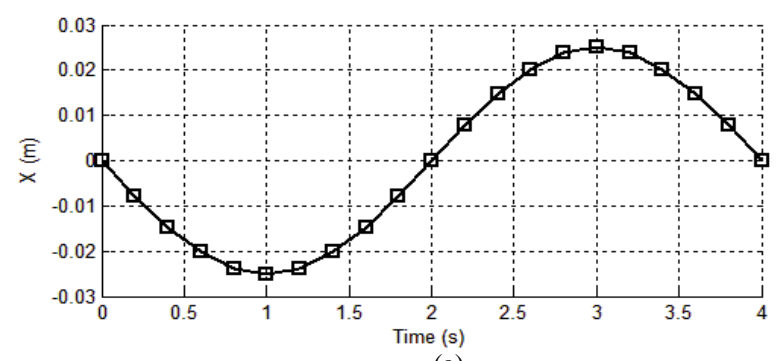

(a)

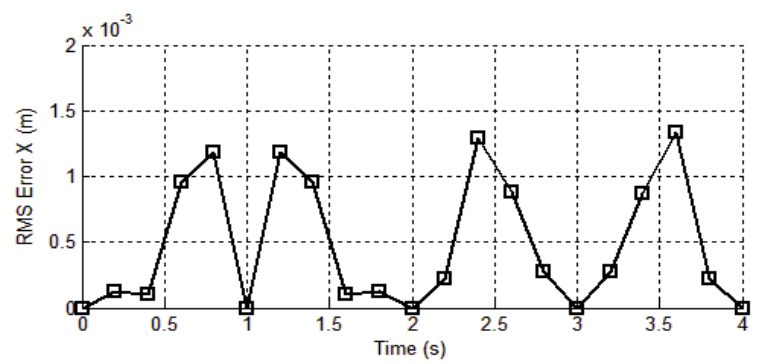

(b)

Fig. 1. (a) A simple pattern for tumor (or implant) movement in $\mathrm{X}$ direction, (b) RMS Error for implant location estimation for the movement pattern in (a). 


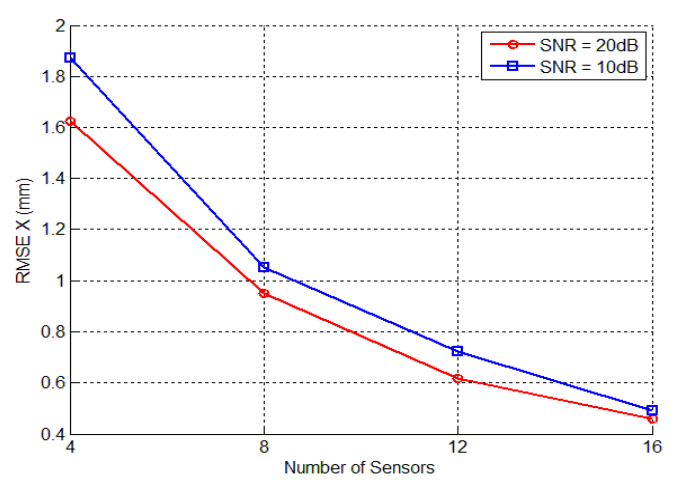

(a)

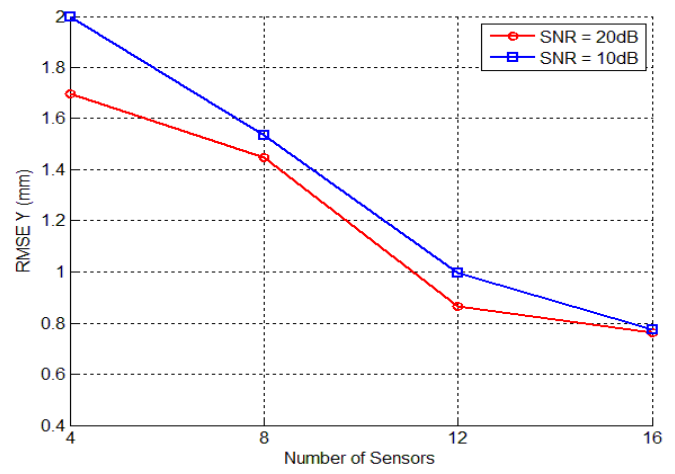

(b)

Fig. 2. RMS Error for $X$ and $Y(\mathrm{~cm})$ versus Number of sensors for two cases $\mathrm{SNR}=0 \mathrm{~dB}$ and $\mathrm{SNR}=10 \mathrm{~dB}$.

\section{REFERENCES}

[1] C. M. Washington and D. T. Leaver, Principles and Practice of Radiation Therapy, 2nd ed. St. Louis, MO: Mosby, 2003.

[2] Wing-Fai Loke, et al., "Magnetic Tracking System for Radiation Therapy," IEEE Transactions on Biomedical Circuits and Systems, Vol. 4, No. 4, Aug 2010.

[3] Andreas W. Rau, et al., "Real-time tumor localization with electromagnetic transponders for image-guided radiotherapy applications," PhD Dissertation, 2009.

[4] S. Dieterich and Y. Suh, Treating Tumors That Move With Respiration. New York: Springer, 2007, ch. Tumor motion ranges due to respiration and respiratory motion characteristics, pp. 3-13.

[5] Amish P. Shah, et al., "Expanding the Use of Real-Time Electromagnetic Tracking in Radiation Oncology," Journal of Appliedclinical Medical Physics, Vol. 12, No. 4, 2011.

[6] Kupelian P, et al. Clinical experience with the Calypso 4D localization system in prostate cancer patients: implantation, tolerance, migration, localization and real time tracking. Int J Radiat Oncol Biol Phys. 2005;63(Suppl 1):S197.

[7] Kupelian, P., et al., "Multi-Institutional Clinical Experience With The Calypso System In Localization And Continuous, Real-Time Monitoring Of The Prostate Gland During External Radiotherapy," International Journal of Radiation Oncology Biology Physics., 2007, 1088-1098.

[8] Willoughby T.R., Kupelian P.A., et al., "Target Localization and Real-time Tracking using the Calypso 4D localization System in Patients with Localized Prostate Cancer," International Journal of Radiation Oncology Biology Physics, 2006, 528-34.

[9] Wing-Fai Loke, et al., "A 0.5-V Sub-mW Wireless Magnetic Tracking Transponder for Radiation Therapy," VLSI Circuits (VLSIC) Symposium, 2011.

[10] Tae-young Choi, et al., "Wireless Magnetic Tracking System for Radiation Therapy," Life Science Systems and Applications Workshop, 2009.

[11] Kindblom J, et al. High precision transponder localization using a novel electromagnetic positioning system in patients with localized prostate cancer. Radiother Oncol. 2009.

[12] A. Plotkin and E. Paperno, "3-D magnetic tracking of a single subminiature coil with a large 2-D array of uniaxial transmitters," IEEE Trans. Magn., vol. 39, no. 5, pp. 3295-3297, Sep. 2003.

[13] E. Paperno and P. Keisar, "Three-dimensional magnetic tracking of biaxial sensors," IEEE Trans. Magn., vol. 40, no. 3, pp. 1530-1536, May 2004.

[14] M. Pourhomayoun, M. Fowler, Z. Jin, "A Novel Method for Medical Implant In-Body Localization," Int. Conf. of the IEEE Engineering in Medicine \& Biology Society (EMBC), 2012.

[15] M. Pourhomayoun and M. Fowler, "Spatial Sparsity Based Emitter Localization," Conf. on Information Sciences and Sys., CISS, 2012.

[16] M. Kawasaki, R. Kohno, "A TOA Based Positioning Technique of Medical Implanted Devices," international Symposium on Medical information \& communication technology, 2009.

[17] K. Sayrafian-Pour, et al., "A statistical path loss model for medical implant communication channels," in Personal, Indoor and Mobile Radio Communications, IEEE 20th International Symposium on, 2009.

[18] M. F. Duarte, Y. C. Eldar, "Structured Compressed Sensing: From Theory to Applications", IEEE Transactions on Signal Process., 2011. 